\title{
Complete Genome Sequence of the Novel Escherichia coli Phage phAPEC8
}

\author{
J. Tsonos, ${ }^{a, b, c}$ E. M. Adriaenssens, ${ }^{a}$ J. Klumpp, ${ }^{\text {d J.-P. Hernalsteens, }}{ }^{\text {b }}$ R. Lavigne, ${ }^{a}$ and H. De Greve ${ }^{c}$ \\ Division of Gene Technology, KU Leuven, Leuven, Belgiumª; Viral Genetics, Vrije Universiteit Brussel, Brussels, Belgiumº; Department of Structural Biology, VIB, Vrije \\ Universiteit Brussel, Brussels, Belgium ${ }^{c}$; and Institute of Food Nutrition and Health, ETH Zurich, Zurich, Switzerland ${ }^{\text {d }}$
}

Bacteriophage phAPEC8 is an Escherichia coli-infecting myovirus, isolated on an avian pathogenic Escherichia coli (APEC) strain. APEC strains cause colibacillosis in poultry, resulting in high mortality levels and important economic losses. Genomic analysis of the 147,737-bp double-stranded DNA phAPEC8 genome revealed that $53 \%$ of the 269 encoded proteins are unique to this phage. Its closest relatives include the Salmonella phage PVP-SE1 and the coliphage rv5, with 19\% and 18\% similar proteins, respectively. As such, phAPEC8 represents a novel, phylogenetically distinct clade within the Myoviridae, with molecular properties suitable for phage therapy applications.

P hage vB_EcoM_phAPEC8 was isolated in Belgium in 2010, from a water sample in the vicinity of a poultry farm, on the avian pathogenic Escherichia coli (APEC) strain CH2 (16). The virulent nature of the phage was demonstrated in vitro by the absence of PFU in an overnight culture of phAPEC8-insensitive $\mathrm{CH} 2$ mutants. PhAPEC8 lyses 11 out of 31 tested APEC isolates and various $\mathrm{CH} 2$ mutants that are resistant against 21 other APEC phages (J. Tsonos, unpublished data). The phage was cultured by the standard soft agar layer technique and purified on a $\mathrm{CsCl}$ gradient (13). Transmission electron microscopy revealed phAPEC8 as a myovirus, similar to PVP-SE1, featuring an isometric head (85 $\mathrm{nm})$ and a contractile tail $(110 \mathrm{~nm})$.

The double-stranded DNA (dsDNA) phAPEC8 genome was sequenced using paired-end reads (Illumina Sequencing Technology). The de novo assembled genome has a G+C content of $39.1 \%$, which is lower than that of its bacterial host $(3,4)$. In total, 269 open reading frames (ORFs), larger than $100 \mathrm{bp}$, were identified by GeneMarkS (2) and NCBI ORF-finder (http://www.ncbi.nlm .nih.gov/projects/gorf/), followed by a manual correction where needed. The gene products were screened for similar proteins using Batch BLASTP at the Greengene facility (http://greengene.uml .edu/programs/Local_Blast.html). Of the 269 presumed proteins, 143 are unique and did not show any similarity with the NCBI nonredundant database. The highest degree of similarity was observed with Salmonella phage PVP-SE1 (51 proteins) and with Escherichia phage rv5 (49 proteins), with 42 gene products shared by all three phages.

Eleven clustered tRNA genes were identified $(9,15)$. One of the regulatory motifs found, TTGACA(N16)TAANAT, is similar to the $\sigma^{70}$ consensus $E$. coli promoter sequence and can therefore be considered a potential host-dependent promoter $(1,7)$. The motif RTAGGGCGGSAATRGTKCCS was always (10 times) present in intergenic regions, systematically upstream of proteins unique to phAPEC8 (10). Eleven rho-independent transcription terminators were identified with ARNold (11).

Of the 37 structural proteins in PVP-SE1, 24 are similar to proteins in phAPEC8, explaining the structural similarity between these phages (14). This morphogenesis cluster of phAPEC8 also encodes a predicted protein with $74 \%$ amino acid identity to the endo- $N$-acetylneuraminidase of the K1-dependent phages K1$\operatorname{dep}(4)$ and K1-dep(1) (accession numbers GU196277.1 and
GU196278.1, respectively). It was already suggested that the sialidase activity of K1-dependent phages provides an increased fitness on capsule-bearing E. coli cells in serum and thus these phages might be more efficient in phage therapy (5). The polysaccharase activity of a predicted protein similar to the colanic acid-degrading protein of phage NST1 (accession number HM214492.1) could allow the phage to penetrate into biofilms (8). Bacterial capsular polysaccharides and colanic acid are both considered virulence factors of $\operatorname{APEC}(6,12)$.

In conclusion, phAPEC 8 can be considered phylogenetically unique within the Myoviridae. The combination of those characteristics, together with the broad host range of phAPEC8, makes this unique Myoviridae an interesting candidate for phage therapeutical applications.

Nucleotide sequence accession number. The genome sequence of phAPEC8 is available under GenBank accession number JX561091.

\section{ACKNOWLEDGMENT}

This research was supported by grant RT 09/8 APECON from the Federal Public Services of Health, Food Chain Safety and Environment (Belgium).

\section{REFERENCES}

1. Bailey TL, et al. 2009. MEME SUITE: tools for motif discovery and searching. Nucleic Acids Res. 37:W202-W208.

2. Besemer J, Lomsadze A, Borodovsky M. 2001. GeneMarkS: a selftraining method for prediction of gene starts in microbial genomes. Implications for finding sequence motifs in regulatory regions. Nucleic Acids Res. 29:2607-2618.

3. Binnewies TT, et al. 2006. Ten years of bacterial genome sequencing: comparative-genomics-based discoveries. Funct. Integr. Genomics 6:165-185.

4. Blattner FR, et al. 1997. The complete genome sequence of Escherichia coli K-12. Science 277:1453-1462.

5. Bull JJ, Vimr ER, Molineux IJ. 2010. A tale of tails: sialidase is key to

Received 31 August 2012 Accepted 1 September 2012

Address correspondence to R. Lavigne, rob.lavigne@biw.kuleuven.be. Copyright $\odot$ 2012, American Society for Microbiology. All Rights Reserved. doi:10.1128/JVI.02374-12 
success in a model of phage therapy against K1-capsulated Escherichia coli. Virology 398:79-86.

6. Ewers C, Janssen T, Wieler LH. 2003. Avian pathogenic Escherichia coli (APEC). Berl. Munch. Tierarztl. Wochenschr. 116:381-395. (In German.)

7. Harley CB, Reynolds RP. 1987. Analysis of E. coli promoter sequences. Nucleic Acids Res. 15:2343-2361.

8. Hughes KA, Sutherland IW, Clark J, Jones MV. 1998. Bacteriophage and associated polysaccharide depolymerase - novel tools for study of bacterial biofilms. J. Appl. Microbiol. 85:583-590.

9. Laslett D, Canback B. 2004. ARAGORN, a program to detect tRNA genes and tmRNA genes in nucleotide sequences. Nucleic Acids Res. 32:11-16.

10. Lavigne R, Sun WD, Volckaert G. 2004. PHIRE, a deterministic approach to reveal regulatory elements in bacteriophage genomes. Bioinformatics 20:629-635.
11. Lesnik EA, et al. 2001. Prediction of rho-independent transcriptional terminators in Escherichia coli. Nucleic Acids Res. 29:3583-3594.

12. Li G, Laturnus C, Ewers C, Wieler LH. 2005. Identification of genes required for avian Escherichia coli septicemia by signature-tagged mutagenesis. Infect. Immun. 73:2818-2827.

13. Sambrook J, Russell DW. 2001. Molecular cloning: a laboratory manual. Cold Spring Harbor Laboratory Press, Cold Spring Harbor, NY.

14. Santos SB, et al. 2011. Genomic and proteomic characterization of the broad-host-range Salmonella phage PVP-SE1: creation of a new phage genus. J. Virol. 85:11265-11273.

15. Schattner P, Brooks AN, Lowe TM. 2005. The tRNAscan-SE, snoscan and snoGPS web servers for the detection of tRNAs and snoRNAs. Nucleic Acids Res. 33:W686-W689.

16. Van den Bosch JF, et al. 1993. Identification of F11 fimbriae on chicken Escherichia coli strains. Infect. Immun. 61:800-806. 